\title{
Cardiovascular, autonomic, and plasma catecholamine responses in unilateral and bilateral carotid artery stenosis
}

Adeola Akinola, Christopher J Mathias, Averil Mansfield, David Thomas, John Wolfe, A N Nicolaides, Thomas Tegos

\begin{abstract}
Objective-To determine impairment of baroreceptor afferent activity, which may affect cardiovascular autonomic function in patients with unilateral and bilateral carotid artery stenosis. Comparison was made with normal subjects and hypertensive patients.

Methods-A series of cardiovascular autonomic function tests along with plasma noradrenaline (norepinephrine) measurements were performed in 46 patients with carotid artery stenosis (CAS); 23 had unilateral and 23 had bilateral stenosis. Comparison was made with 21 hypertensive patients (with a similar degree of raised blood pressure), and 27 normal subjects.

Results-Over a third of patients with unilateral and bilateral CAS had postural hypotension. Heart rate did not rise appropriately in CAS despite the postural fall in blood pressure. Pressor responses in CAS were preserved. Heart rate responses to respiratory stimuli were attenuated. Plasma noradrenaline concentrations rose normally during head up tilt. Conclusion-A substantial proportion with CAS had postural hypotension and attenuated heart rate responses. This was not due to sympathetic vasoconstrictor or cardiac parasympathetic failure, thus suggesting impaired afferent baroreceptor activity, probably secondary to involvement of the carotid sinus. Cardiovascular autonomic testing and assessment of postural hypotension and factors enhancing it may be of clinical relevance in such patients, especially as the symptoms of postural hypotension overlap with those due to thromboembolism.
\end{abstract}

(F Neurol Neurosurg Psychiatry 1999;67:428-432)

Keywords: catecholamine responses; carotid artery stenosis

The baroreceptor reflex plays a key part in the beat by beat regulation and maintenance of arterial blood pressure. Its afferents are located mainly in the carotid sinus and aortic arch. A major factor is their stimulation by distension, which may be reduced in disease, commonly as a result of atherosclerosis. ${ }^{12}$ In patients with carotid artery stenosis (CAS), there may be substantial impairment of baroreceptor afferent activity, especially if it is bilateral. In experimental studies, reduction of afferent carotid sinus nerve activity has been associated with development of hypertension. ${ }^{3}$ The reverse, a fall in blood pressure, may also occur because of such impairment, and may account for reports in which hypotension has been associated with ingestion of food, postural change, and angiography. ${ }^{4}$

Most previous studies have concentrated on baroreflex assessment and on mechanisms of hypertension in patients with CAS. ${ }^{6-8}$ There have been no detailed studies using physiological testing as utilised in patients with autonomic failure, to determine responses in those with clearly defined unilateral and bilateral CAS. We describe such studies using comparison with age and sex matched normal subjects. As most patients with CAS were hypertensive and taking drugs, comparisons were made with a group with a similar degree of hypertension who were taking similar antihypertensive drugs, but in whom there was no evidence of carotid artery stenosis.

\section{Patients and methods}

PATIENTS

Forty six patients with CAS (age range 58-81 years) were studied. Twenty three patients (13 men and 10 women; mean age 69.2 (SD 6.5) years, had unilateral (uCAS), and 23 (16 men and seven women; mean age 68.6 (SD 6.9) years) had bilateral (bCAS) CAS. The criteria for clinically significant CAS was based on $>80 \%$ CAS using duplex sonography and carotid angiography (intravenous/intra-arterial digital subtraction angiography). In most cases of uCAS the contralateral artery had a $40-50 \%$ stenosis. A history of transient ischaemic attacks was obtained in all patients. This consisted of ipsilateral monocular visual loss or contralateral homonymous visual field loss in about $50 \%$, and contralateral paresis, sensory loss or clumsiness in the hand, arm, face, or leg; aphasia; or dysarthria in the rest. In addition, $20 \%$ had symptoms of posturally related dizzinness. None, however, had clinical evidence of a residual neurological deficit. Brain CT was normal in all. The majority with CAS had a history of hypertension and at the time of study $70 \%$ were taking antihypertensive drugs. Their drug therapy included angiotensin converting enzyme inhibitors (30\%), calcium channel blockers (46\%), diuretics $(30 \%)$, and $\beta$-blockers (30\%). These were stopped for 24 hours before testing. This was thought to be a safe period for drug withdrawal in these 
Table 1 Blood pressure and heart rates in controls (NT-c), hypertensive patients (HT-c), and patients with unilateral $(u C A S)$ or bilateral $C A S(b C A S)$

\begin{tabular}{|c|c|c|c|c|c|c|}
\hline & & & Controls $(N T-c)$ & $H T-c$ & $u C A S$ & $b C A S$ \\
\hline \multirow{2}{*}{\multicolumn{2}{|c|}{ Supine }} & $\mathrm{BP}$ & $137(7) / 82(2)$ & $153(5)+/ 85(2)$ & $160(10) \dagger / 76(4)$ & $171(7)+/ 88(5)$ \\
\hline & & HR & $74(2)$ & $68(2)$ & $67(4)$ & 67 (3) \\
\hline \multirow[t]{4}{*}{ Head up tilt } & 2 Minutes & $\mathrm{BP}$ & $131(6) / 86(3)$ & $156(4) / 90(3)$ & $135(13)^{\star} \ddagger / 70(5)$ & $149(8)^{\star \star} \ddagger / 82(4)$ \\
\hline & & HR & $76(2)^{\star}$ & $70(3)^{\star}$ & $70(3)^{\star}$ & $69(3)$ \\
\hline & 5 Minutes & $\mathrm{BP}$ & $135(6) / 86(2)$ & $155(5) / 88(3)$ & $129(9)^{\star} \ddagger \ddagger / 72(5)$ & $142(6)^{\star \star \star} \ddagger \ddagger \neq / 79$ (3) \\
\hline & & HR & $79(2)^{\star}$ & 71 (2) & $70(4)$ & 69 (3) \\
\hline \multirow[t]{2}{*}{ Supine } & & $\mathrm{BP}$ & $132(4) / 79(2)$ & $156(3) \S / 86(3)$ & $161(8) \S / 84(4)$ & $162(6) \$ / 89(3)$ \\
\hline & & HR & $68(2)$ & $70(3)$ & $72(3)$ & $70(3)$ \\
\hline \multirow[t]{4}{*}{ Standing } & 2 Minutes & $\mathrm{BP}$ & $127(6) / 81(2)$ & $155(4) / 86(3)$ & $126(3)^{\star \star \star} \ddagger / 76(4)$ & $127(6)^{\star \star \star} \ddagger / 77$ (3) \\
\hline & & HR & $79(3)^{\star \star}$ & $72(2)$ & $78(4)^{\star}$ & $74(3)$ \\
\hline & 5 Minutes & $\mathrm{BP}$ & $136(4) / 84(2)$ & $154(5) / 88(3)$ & $135(8)^{\star \star \star} \neq \ddagger / 74(2)$ & 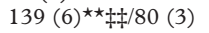 \\
\hline & & HR & $79(2)^{\star \star}$ & $71(2)$ & $77(4)$ & $72(3)$ \\
\hline
\end{tabular}

Values are expressed as means (SEM); $\mathrm{BP}=$ blood pressure; $\mathrm{HR}=$ heart rate.

Significant changes from resting value: ${ }^{\star} \mathrm{p}<0.05 ;{ }^{\star \star} \mathrm{p}<0.001 ;{ }^{\star \star \star} \mathrm{p}<0.0001$

Significant differences in baseline BP in NT-c compared with HT-c, uCAS, and bCAS: $+\mathrm{P}<0.05$

Significant differences in BP change in HT-c during head-up tilt/standing compared with uCAS and bCAS: $\ddagger \mathrm{p}<0.001 ; \ddagger \neq \mathrm{p}<0.0001$. No significant BP/HR differences between uCAS and bCAS.

patients. None had disorders such as diabetes mellitus that could impair interpretation of data.

Twenty seven age matched normal patients (NT-c; age range 60-69 years) were studied as controls. However, because of the presence of hypertension and drug treatment in CAS, comparison was also made with 21 aged matched hypertensive patients (HT-c, 12 men and nine women; mean age 66.5 (SD 8.6) years) with similar levels of blood pressure but in each of whom Doppler ultrasound scanning excluded CAS. They were on similar drug therapy; this included angiotensin converting enzyme inhibitors (30\%), calcium channel blockers (40\%), diuretics (40\%), and $\beta$-blockers (20\%). As in the patients with CAS, drugs were stopped 24 hours before testing.

The study was performed with the understanding and consent of each subject and was approved by the ethics committee of St Mary's Hospital, London.

\section{METHODS}

All studies were performed in a clinical investigation laboratory after an overnight fast. Continuous non-invasive recording of beat by beat finger blood pressure and heart rate was by the Finapres (Ohmeda, 2300 Finapres), in addition to intermittent brachial blood pressure measured with an automated sphygmomanometer (Dinamap). Continuous ECG monitoring was obtained from two electrodes placed on the chest. A cannula was inserted into a vein in the antecubital fossa for blood sampling. Physiological tests of autonomic function were performed using previously described techniques. ${ }^{9}$ The tests were performed in the order outlined below. Patients rested in between each test until blood pressure and heart rate returned to baseline. A brief description of the tests follows:

\section{Postural tests}

After 10 minutes of supine rest, the subject was tilted head up to $45^{\circ}$ on a tilt table. Blood pressure and heart rate were measured after 2, 5, and 10 minutes. On a separate occasion after basal measurements, the subject stood up unaided for 5 minutes.

\section{Valsalva manoeuvre}

The Valsalva manoeuvre was performed by blowing through a mouthpiece attached to a manometer and maintaining a pressure of 40 $\mathrm{mm} \mathrm{Hg}$ for 15 seconds. The Valsalva ratio was calculated from the ratio of the longest R-R interval after the manoeuvre (phase 4 ) to the shortest $\mathrm{R}-\mathrm{R}$ interval during the manoeuvre (phase 2).

\section{Pressor tests}

Isometric exercise-The subject gripped a sphygmomanometer cuff inflated to $20 \mathrm{~mm} \mathrm{Hg}$ with their dominant hand at one third of maximal voluntary contraction force for 2 minutes. Blood pressure was measured at rest and at the end of handgrip.

Cutaneous cold (cold pressor test) - The hand, up to the wrist, was immersed in ice slush (temperature about $4^{\circ} \mathrm{C}$ ) for 2 minutes. Blood pressure and heart rate were measured before and after the test.

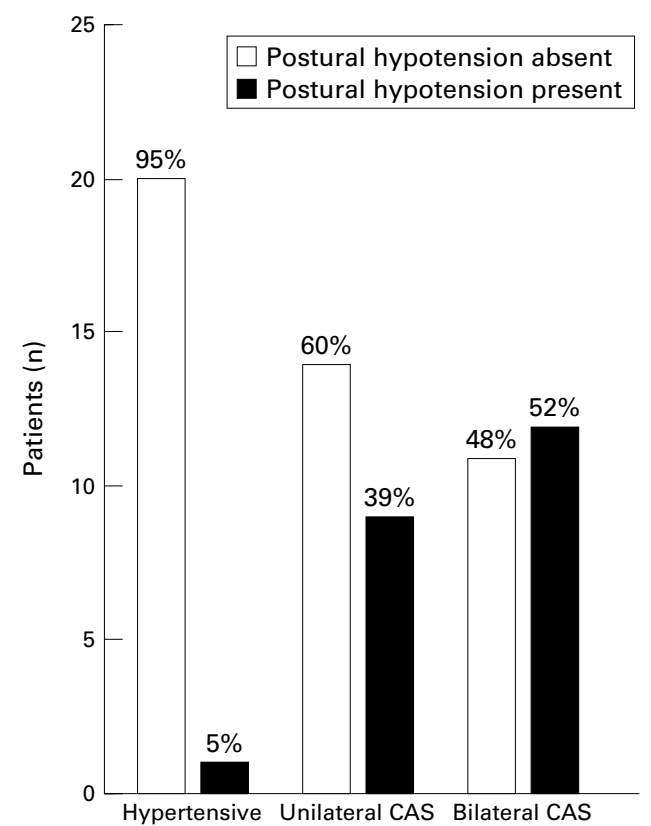

Total number/percentages of hypertensive, unilateral CAS, and bilateral CAS patients with and without postural hypotension during head up tilt and on standing. 
Table 2 Summary of autonomic function tests in controls, hypertensive patients, and patients with unilateral or bilateral carotid artery stenosis

\begin{tabular}{|c|c|c|c|c|}
\hline & $\begin{array}{l}\text { Controls } \\
n=27\end{array}$ & $\begin{array}{l}\text { Hypertensive } \\
n=21\end{array}$ & $\begin{array}{l}\text { Unilateral } \\
C A S n=23\end{array}$ & $\begin{array}{l}\text { Bilateral CAS } \\
n=23\end{array}$ \\
\hline \multicolumn{5}{|l|}{ Orthostatic hypotension: } \\
\hline On tilt or standing & $0(0 \%)$ & $1(5 \%)$ & $9(39 \%)$ & $12(52 \%)$ \\
\hline \multicolumn{5}{|l|}{ Valsalva manoeuvre: } \\
\hline Valsalva ratio (VR) & $1.62(0.6)$ & $1.31(0.05)$ & $1.14(0.03)$ & $1.13(0.03)$ \\
\hline Abnormal VR & & $12(57 \%)$ & $16(69 \%)$ & $16(69 \%)$ \\
\hline \multicolumn{5}{|l|}{ Pressor responses: } \\
\hline \multicolumn{5}{|l|}{ Isometric exercise } \\
\hline Change in $\mathrm{BP}(\mathrm{mm} \mathrm{Hg})$ & $17(3) / 11(2)$ & $16(3) / 9(2)$ & $16(2) / 13(2)$ & $16(4) / 15(4)$ \\
\hline \multicolumn{5}{|l|}{ Cold } \\
\hline Change in $\mathrm{BP}(\mathrm{mm} \mathrm{Hg})$ & $15(2) / 10(2)$ & $13(2) / 12(2)$ & $15(2) / 8(2)$ & $11(3) / 6(2)$ \\
\hline Diminished response & & $0(0 \%)$ & $0(0 \%)$ & $1(4 \%)$ \\
\hline Exaggerated response & & $5(24 \%)$ & $5(22 \%)$ & $5(22 \%)$ \\
\hline \multicolumn{5}{|l|}{ Sinus arrhythmia: } \\
\hline HR difference (beats/min) & $12(1)$ & $10(1)$ & $9(1)$ & $6(1)$ \\
\hline
\end{tabular}

$\mathrm{VR}=$ Valsalva ratio; $\mathrm{BP}=$ blood pressure; $\mathrm{HR}=$ heart rate.

Mental arithmetic - Patients performed serial subtractions for 2 minutes with measurements before and at the end of the test.

Deep breathing test

The subject breathed deeply six times in 1 minute while supine. The difference between the maximum and minimum heart rates in an individual respiratory cycle was calculated and expressed as the mean of the differences in six successive cycles.

Plasma catecholamine measurements Blood samples for measurement of plasma noradrenaline (norepinephrine), adrenaline (epinephrine), and dopamine were taken at rest and during head up tilt. Plasma concentrations were measured by high performance liquid chromatography with an electrochemical detector. ${ }^{10}$ The intra-assay and interassay coefficients of variation were $3.1 \%$ and $4.6 \%$ (respectively) for noradrenaline, $4.6 \%$ and 5.1 $\%$ for adrenaline, and $6.4 \%$ and $5.8 \%$ for dopamine.

STATISTICS

Results are expressed as means (SEM). Orthostatic hypotension was defined as a fall $>20$ $\mathrm{mm} \mathrm{Hg}$ systolic pressure while standing or during head up tilt. ${ }^{911}$ Statistical analyses were performed using student's independent $t$ tests for comparing changes within subject group, and analysis of variance (ANOVA), student's $t$ tests, and $\chi^{2}$ test for comparing changes between subject groups (Minitab Data Analysis Software, Release 7. 1, State College, PA, USA); a p value $<0.05$ was considered significant. Non-significant changes are expressed as NS.

\section{Results}

Orthostatic tests

Pretilt supine blood pressure was higher in HT-c, uCAS, and bCAS than in NT-c. With head up tilt, blood pressure did not fall in any subject in the NT-c and HT-c groups. Orthostatic hypotension (a fall of $20 \mathrm{~mm} \mathrm{Hg}$ or more in systolic blood pressure) occurred in nine (39\%) uCAS (by 31 (8) $\mathrm{mm} \mathrm{Hg}$; $\mathrm{p}<0.005)$ and 11 (48\%) bCAS (by 29 (5) $\mathrm{mm} \mathrm{Hg}$; $\mathrm{p}<0.0002)$. On standing, blood pressure fell in one (5\%) HT-c, eight (35\%) uCAS (by 26 (4) $\mathrm{mm} \mathrm{Hg}$. p<0.0001) and in $10(43 \%)$ bCAS (by 28 (4); $p<0.0001$ ) (table 1, fig). Postural hypotension in CAS was sustained. It occurred with both head up tilt and standing. In addition, eight CAS with postural hypotension were studied both before and after carotid endarterectomy. There was no change in autonomic function in six, one became normotensive without postural hypotension postoperatively, and one hypertensive patient developed postural hypotension which necessitated a marked reduction in antihypertensive therapy.

Supine pretilt heart rate was similar in NT-c, HT-c, uCAS, and bCAS. With head up tilt, heart rate increased similarly in NT-c, HT-c, uCAS, and bCAS. In uCAS and bCAS with postural hypotension the increase in heart rate with tilt was attenuated (table 1).

\section{Valsalva manoeuvre}

The Valsalva ratio (VR) in NT-c was 1.62 (0.06). It was lower in HT-c (1.31 (0.05); $\mathrm{p}<0.0004)$. Levels in uCAS $(1.14(0.03))$ and bCAS (1.13 (0.03)) were lower than HT-c (each $\mathrm{p}<0.005)$. An abnormal VR occurred in 12 (57\%) HT-c, 16 (69\%) uCAS, and 16 (69\%) bCAS compared with NT-c (table 2). All CAS with postural hypotension had an abnormal Valsalva ratio.

\section{Response to pressor stimuli}

With isometric exercise, blood pressure and heart rate rose similarly in each group (table 3 ). With cutaneous cold, blood pressure rose in each group to the same extent. Heart rate remained unchanged in all groups (table 3). With mental arithmetic, blood pressure and heart rate rose similarly in each group. Responses to at least one pressor stimulus were exaggerated in five (24\%) HT-c, five (22\%) uCAS, and five $(22 \%)$ bCAS. Diminished responses occurred in one (4\%) bCAS (tables 2 and 3$)$.

Table 3 Changes in systolic (SBP) and diastolic (DBP) blood pressure and heart rate (HR) during isometric exercise and cold pressor test in controls, hypertensive patients, and patients with, unilateral or bilateral CAS

\begin{tabular}{|c|c|c|c|c|c|c|c|c|c|}
\hline & \multicolumn{3}{|c|}{ Isometric exercise } & \multicolumn{3}{|c|}{ Cutaneous cold } & \multicolumn{3}{|c|}{ Mental arithmetic } \\
\hline & $S B P$ & $D B P$ & $H R$ & $S B P$ & $D B P$ & $H R$ & $S B P$ & $D B P$ & $H R$ \\
\hline Controls & $17(3)^{\star \star \star}$ & $11(2)^{\star \star \star}$ & $4(1)^{\star}$ & $15(2)^{\star \star \star}$ & $10(2)^{\star}$ & $1(10$ & $13(3)^{\star \star \star}$ & $9(2)^{\star}$ & $8(2)^{\star}$ \\
\hline Hypertensive & $16(3)^{\star \star \star}$ & $9(2)^{\star \star \star}$ & $5(1)^{\star}$ & $13\left(20^{\star \star \star}\right.$ & $12(2)^{\star \star \star}$ & $3(1)$ & $6(3)^{\star \star \star}$ & $16(2)^{\star}$ & $4(2)^{\star}$ \\
\hline Unilateral CAS & $16(2)^{\star \star \star}$ & $13(2)^{\star \star \star}$ & $4(1)^{\star}$ & $15(2)^{\star \star \star}$ & $8(2)^{\star}$ & $-1(1)$ & $17(4)^{\star \star \star}$ & $11(3)^{\star}$ & $6(2)^{\star}$ \\
\hline Bilateral CAS & $16(4)^{\star \star \star}$ & $15(4)^{\star \star \star}$ & $5(2)^{\star}$ & $11(3)^{\star \star \star}$ & $6(2)^{\star}$ & $2(1)$ & $13(3)^{\star \star \star}$ & $9(4)^{\star}$ & $6(2)^{\star}$ \\
\hline
\end{tabular}

Values are expressed as means (SEM). Significant changes from resting value: ${ }^{\star} \mathrm{p}<0.05 ;{ }^{\star \star} \mathrm{p}<0.001 ;{ }^{\star \star \star} \mathrm{p}<0.0001$. No significant differences between controls, hypertensive patients, and patients with unilateral or bilateral CAS. 
Deep breathing

With deep breathing, inspiratory-expiratory heart rate differences were similar in NT-c (12 (1)), HT-c (10 (1)), and uCAS (9 (1)), but was lower in bCAS (6 (1)); p<0.001, table 2).

\section{Plasma catecholamines}

Resting venous plasma noradrenaline concentrations were 296 (45) pg/ml, 305 (36) pg/ml, 395 (38) $\mathrm{pg} / \mathrm{ml}$, and 353 (30) $\mathrm{pg} / \mathrm{ml}$ in NT-c, HT-c, uCAS, and bCAS respectively; there was no difference between the groups. With head up tilt noradrenaline concentrations rose similarly in all groups (to 456 (72) pg/ml, 411 (51) $\mathrm{pg} / \mathrm{ml}, 565$ (44) $\mathrm{pg} / \mathrm{ml}$, and 444 (48) $\mathrm{pg} / \mathrm{ml}$ respectively). Resting venous adrenaline concentrations were 43 (8) $\mathrm{pg} / \mathrm{ml}, 41$ (7) pg/ml, 47 (7) $\mathrm{pg} / \mathrm{ml}$, and 41 (8) $\mathrm{pg} / \mathrm{ml}$ respectively; concentrations did not change with tilt. Mean resting plasma dopamine concentrations were below detectable levels of $5 \mathrm{pg} / \mathrm{ml}$ in each group.

\section{Discussion}

This study indicates that the cardiovascular responses to certain physiological stimuli were impaired in patients with uCAS and bCAS compared with hypertensive patients and normotensive controls. Of note was the large proportion of patients with CAS who had definite postural hypotension (about $30 \mathrm{~mm} \mathrm{Hg}$ systolic pressure) on head up tilt and standing. Postural hypotension has not been reported in this group of patients except for case reports in which transient ischaemic attacks were probably due to hypotension. ${ }^{4}{ }^{12}$ In none was there a complete evaluation of postural and other cardiovascular autonomic responses. The reasons for the high incidence of postural hypotension in CAS warrant discussion. One may have been their antihypertensive therapy. Medication was stopped 24 hours before the study; this would have reduced but not eliminated residual effects. However, most antihypertensive drugs currently used are not known to cause postural hypotension. Furthermore, in the hypertensive group (without CAS), on similar therapy, postural hypotension did not occur.

A further reason for postural hypotension was sympathetic vasoconstrictor failure, as occurs in patients with primary autonomic failure, with central or peripheral sympathetic denervation. ${ }^{9}$ This, however, was unlikely as responses to pressor stimuli (that reflect sympathetic efferent activity) were normal or even exaggerated in CAS. Furthermore, the plasma noradrenaline response induced by head up tilt (as a biochemical measure of efferent activity) was preserved. Therefore, this raises the question of whether postural hypotension was induced by impairment of afferent baroreceptor function as may be expected, especially in bCAS. However, there was no difference between the degree or extent of postural hypotension in uCAS and bCAS. It may be that in uCAS, the dominant afferent was impaired; alternatively, despite the absence of clinically significant contralateral artery stenosis, there may have been sufficient atheroma to reduce distensibility and thus affect afferent baroreceptor activity. Thus, pathophysiologically, the patients with uCAS may have been similar to those with bCAS; this may account for a similar functional deficit.

The responses to the other physiological tests provided further information on the possible autonomic abnormalities in the CAS patients. The responses to various pressor tests indicated that these were similar in the four groups studied. However, further analysis, in comparison with the normotensive subjects, suggested enhanced pressor responsiveness in the hypertensive patients, with a similar proportion of uCAS and bCAS exhibiting such responses. This again may reflect impaired baroreflex activity. In the hypertensive patients, factors such as the resetting of the baroreceptors at a higher threshold pressure, or reduced sensitivity have been postulated ${ }^{13-16}$; this may have accounted for their exaggerated responses, with impaired baroreflex afferents causing the abnormal responses in uCAS and bCAS. ${ }^{17}$

Plasma noradrenaline concentrations may reflect sympathetic vasoconstrictor activity; however, many factors influence noradrenaline concentrations in plasma and this is an indirect measure of activation of sympathetic efferents. The plasma noradrenaline concentrations in the resting supine state were similar in each of the groups, suggesting a similar degree of resting sympathetic tone. With head up tilt the concentrations rose similarly in each group, again confirming their ability to increase sympathetic nerve activity. Plasma noradrenaline concentrations rose in patients with CAS.

The heart rate responses to various tests were abnormal, especially in bCAS. The rise in heart rate with postural change was reduced. Abnormalities of heart rate were also recorded during the Valsalva manoeuvre, which reflects overall baroreflex changes. With deep breathing however, the responses seemed preserved, except in patients with bCAS. This makes impairment of vagal efferents less likely and would be in favour of the afferent abnormality accounting for the abnormal responses.

Overall, therefore, the findings suggest that cardiac parasympathetic efferent pathways were relatively unaffected in uCAS and bCAS. This again is consistent with impairment mainly of the afferent limb of the baroreflexpossibly that is, within the carotid sinus region; we recognise that there are other afferents that influence cardiovascular function and that these may remain operative in these patients.

This study, despite the limitations (that could not be avoided), of studying patients on antihypertensive drug therapy, indicates that postural hypotension occurs in a substantial number of patients with uCAS and bCAS compared with hypertensive patients on similar drugs. This is of clinical relevance to these patients. The key symptoms of postural hypotension are mainly the result of cerebral hypoperfusion and include dizziness, visual disturbances, impaired cognition, and syncope ${ }^{18}$; thus these may mimic symptoms resulting from thromboembolism. It is not 
clear if antihypertensive therapy may worsen postural hypotension and thus cause more symptoms. Although in this study we report the responses to head up tilt and standing, it is possible that the effects of various stimuli in daily life, ranging from food ingestion to mild exercise, that are now recognised as unmasking or enhancing hypotension, ${ }^{19}{ }^{20}$ will need to be considered further, as these stimuli may increase the tendency to hypotension where there is autonomic impairment.

In conclusion, therefore, our studies utilising a series of cardiovascular autonomic function tests, indicate that postural hypotension occurs in a substantial number of patients with both uCAS and bCAS. This does not seem to be due to sympathetic efferent failure but is probably due to abnormalities resulting from impaired baroreceptor afferents, presumably in the region of the carotid sinus. Postural hypotension may increase the susceptibility to cerebral hypoperfusion, and contribute further to morbility and mortality in CAS. This needs to be determined in future studies.

We thank Dr H S Chang, Mrs Laura Judd, and Miss Laura Watson for their assistance, and the Stroke Association for their support.

1 Winson M, Heath D, Smith P. Extensibility of the human carotid sinus. Cardiovasc Res 1974;8:58-64.

2 Sleight P. Neurophysiology of the carotid sinus receptors in normal and hypertensive animals and man. Cardiology 1976;61 (suppl):31-45.

3 Dehn TCB, Angell JE. Long term effect of carotid endarterectomy on carotid sinus baroreceptor function and blood ectomy on carotid sinus baroreceptor function
pressure control. Br ₹ Surg 1987;74:997-1000.

pressure control. Brf Surg 1987;74:997-1000.
4 Ruff RL, Taiman WT, Tetito F. Transient ischaemic attacks associated with hypotension in hypertensive patients with associated with hypotension in hypertensive
carotid artery stenosis. Stroke 1981;12:353-5.

5 Karotid artery stenosis. Stroke 1981;12:353-5. attack caused by post-prandial hypotension. Stroke 1994; 25:511-13
6 Skydell JL, Machleder HI, Baker JD, et al. Incidence and mechanisms of post-carotid endarterectomy hypertension. Arch Surg 1987;122:1153-5.

7 Hirschl M, Kundi M, Hirsch MM, et al. Blood pressure responses after carotid artery surgery: relationship to post operative baroreceptor sensitivity. Am f Med 1993;33:4638.

8 Hirschl M, Kundi M, Blazek G. Five year follow up of patients after thromboendarectomy of the internal carotid artery. Relevance of baroreceptor sensitivity. Stroke 1996; 27:1167-72.

9 Mathias CJ, Bannister R. Investigation of autonomic disorders. In: R Bannister, CJ Mathias, eds. Autonomic failure. A textbook of clinical disorders of the autonomic nervous system. 4th ed. Oxford: Oxford University Press, 1999:169-95.

10 Mathias CJ, Bannister R, Cortelli P, et al. Clinical, autonomic and therapeutic observations in two siblings with postural hypotension and sympathetic failure due to in in ility to synthesize noradrenaline from dopamine because of a deficiency of dopamine beta hydroxylase. $O F$ Med 1990; New Series 75;278:617-33.

11 Consensus statement. Definition of orthostatic hypotension, pure autonomic failure and multiple system atrophy. Clin Auton Res 1996;6:125-6.

12 Dobkin $\mathrm{BH}$. Orthostatic hypotension as a risk factor for symptomatic occlusive cerebrovascular disease. Neurology 1989;39:30-4.

13 Bristow JD, Honour AJ, Pickering GW, et al. Diminished baroreflex in high blood pressure. Circulation 1969;39:4853.

14 Randall OS, Esler MD, Bulloch GF, et al. Relationship of age and blood pressure to baroreflex sensitivity and arterial compliance in man. Clinical Science and Molecular Medicine 1976;51:357-60S

15 Vlachakis ND, Mendlowitz M, De Gusman D, et al. Diminished baroreceptor sensitivity of elderly hypertensives. Possible role of atherosclerosis. Atherosclerosis 1976;24:243-9.

16 Shimada K, Kitazumi T, Ogura A, et al. Differences in ageindependent effects of blood pressure on baroreflex inderitivity between normal and hypertensive patients. Clin sensitivity between n
Sci 1986;70:489-94.

17 Ille 0, Woimant F, Pruna A, et al. Hypertensive encephalopathy after bilateral carotid endarterectomy. Stroke 1995;26: 488-91.

18 Mathias CJ. Orthostatic hypotension-causes, mechanisms and influencing factors. Neurology 1995;45 (suppl 5):S611.

19 Mathias CJ, Holly E, Armstrong E, et al. The influence of food on postural hypotension in three groups with chronic autonomic failure: clinical and therapeutic implications. $\mathcal{f}$ autonomic failure: clinical and therapeutic imp

20 Smith GDP, Mathias CJ. Postural hypotension enhanced by exercise in patients with chronic autonomic failure. $Q \mathcal{F}$ Med 1995;88:251-6. 